\title{
Comparison of the Freiburg and Charlson Comorbidity Indices in Predicting Overall Survival in Elderly Patients with Newly Diagnosed Multiple Myeloma
}

\author{
Sung Min Kim, Moon Jin Kim, Hyun Ae Jung, Kihyun Kim, Seok Jin Kim, Jun Ho Jang, \\ Won Seog Kim, and Chul Won Jung
}

Division of Hematology-Oncology, Department of Medicine, Samsung Medical Center, School of Medicine, Sungkyunkwan University, 81 Irwon-ro, Gangnam-gu, Seoul 135-710, Republic of Korea

Correspondence should be addressed to Kihyun Kim; kihyunkimk@gmail.com

Received 25 March 2014; Revised 29 May 2014; Accepted 18 June 2014; Published 10 July 2014

Academic Editor: Dong Soon Lee

Copyright (C) 2014 Sung Min Kim et al. This is an open access article distributed under the Creative Commons Attribution License, which permits unrestricted use, distribution, and reproduction in any medium, provided the original work is properly cited.

\begin{abstract}
Multiple myeloma occurs primarily in elderly patients. Considering the high prevalence of comorbidities, comorbidity is an important issue for the management of myeloma. However, the impact of comorbidity on clinical outcomes has not been fully investigated. We retrospectively analyzed patients with newly diagnosed myeloma. Comorbidities were assessed based on the Charlson comorbidity index (CCI) and the Freiburg comorbidity index (FCI). The CCI is a summary measure of 19 comorbid conditions. FCI is determined by performance status, renal impairment, and lung disease. This study included 127 patients with a median age of 71 years. Approximately half of the patients had additional disorders at the time of diagnosis, and diabetes mellitus was the most frequent diagnosis (18.9\%). The most significant factors for prognosis among patient-related conditions were a history of solid cancer and performance status (ECOG $\geq 2)$. The FCI score was divided into 3 groups $(0,1$, and 2-3), and the CCI score was divided into 2 groups $(2-3$ and $\geq 4)$. FCI was a strong prognostic tool for OS $(P>0.001)$ and predicted clinical outcome better than CCI $(P=0.059)$. In conclusion, FCI was more useful than CCI in predicting overall survival in elderly patients with myeloma.
\end{abstract}

\section{Introduction}

Multiple myeloma (MM) is a hematologic malignancy of plasma cells that results in bone destruction, marrow failure, and renal impairment. The median age at the time of diagnosis is 70 years, with $36 \%$ of patients younger than 65 years, $27 \%$ aged 65 to 74 years, and $37 \%$ older than 75 years [1]. Considering the increasing life expectancy of the general population, the number of geriatric patients affected by MM is expected to increase over time.

Risk stratification of myeloma using the international staging system (ISS) and host factors such as age, performance status, and comorbidities are thought to be important for determining prognosis and choosing treatment options [2-4]. In 2011, Palumbo et al. suggested that appropriate screening for age ( $>75$ years) and vulnerability, in addition to assessment of cardiac, pulmonary, renal, hepatic, and neurological functions, at the start of therapy allows treatment strategies to be individualized and drug doses to be tailored to improve tolerability and optimize efficacy [5]. Their study emphasized that elderly MM patients are more susceptible to side effects and are often unable to tolerate full drug doses. For these patients, lower-dose intensity regimens improve the safety profile and thus optimize the treatment outcome. However, this study was not based specifically on MM because there were few data on the impact of vulnerability on outcomes in MM patients [5]. Nonetheless, in general, several studies have reported problems related to comorbidity and cancer treatment in elderly patients [6-10].

Kleber et al. developed the Freiburg comorbidity index (FCI) to assess patient-related conditions as a risk factor for MM. The FCI is composed of three comorbidity factors: renal impairment, moderate to severe lung disease, and performance status. Interestingly, the FCI showed strong clinical relevance for overall survival (OS) and progression-free survival (PFS). Moreover, compared with other comorbidity indices, 
such as the Charlson comorbidity index (CCI), hematopoietic cell transplantation-specific comorbidity index (HCT$\mathrm{CI}$ ), Kaplan-Feinstein (KF), and Satariano index (SI), FCI was better able to stratify risk in patients with MM [11].

Although CCI is a widely used tool for assessing comorbidity in malignancy, this comorbidity index is complicated and difficult to apply. Moreover, there is no proven cut-off value that divides patients into low- or high-risk groups. As a result, several studies have determined their own cutoff values [11-16]. For example, Offidani et al. suggested a vulnerability score consisting of performance status and comorbidity score of CCI 0 or $\geq 1$ [15].

Because of the increased incidence of multiple myeloma with aging and the fact that elderly patients have more comorbidity than younger patients, in the present study, we assessed comorbidities at diagnosis, the impact of host factors on OS, and compared CCI and FCI as prognostic factors in newly diagnosed elderly patients with MM.

\section{Methods}

2.1. Study Design. This study was a retrospective, singlecenter case series. OS was calculated as the time from diagnosis to death from any cause. Adverse events were graded according to the National Cancer Institute Common Toxicity Criteria (NCI-CTC) version 4.0. This study was reviewed and approved by the Institutional Review Board.

2.2. Patients. A total of 127 consecutive patients aged 65 years and older who were newly diagnosed with symptomatic MM at the Samsung Medical Center, Seoul, South Korea, between January 1, 1999, and June 30, 2011, met the inclusion criteria for this study. We excluded patients with amyloidosis, those who were suitable for autologous stem cell transplantation (ASCT), and those who were lost to follow-up within 6 months from the time of diagnosis due to any cause except death. The last follow-up date was March 31, 2013.

2.3. Charlson Comorbidity Index (CCI). The CCI (Table 1) is a summary measure of 19 comorbid conditions that are each weighted from 1 to 6 based on disease severity. This measure provides a total score ranging from 0 to 37 [17]. Information on comorbidity was extracted from a detailed review of each patient's medical records and laboratory values at the time of diagnosis. In the original study, MM was included in the classification of lymphoma for convenience. In this study, lymphoma was defined, as in the original study, except that the definition did not include myeloma.

In addition, according to the original study, each decade of age over 40 would add 1 point to the risk value (i.e., 50 years $=+1$ point), and the age point would be added to the score of the comorbidity index. In this study, we used the method described by Kleber et al., which adds the age point to the CCI score [11].

2.4. Freiburg Comorbidity Index (FCI). Renal impairment is defined as estimated glomerular filtration rate (eGFR) $\leq 30 \mathrm{~mL} / \mathrm{min} / 1.73 \mathrm{~m}^{2}$, based on the modification of diet in renal disease (MDRD) study equation [18]. Poor performance status is based on a Karnofsky performance status (KPS) score $\leq 70$. Moderate or severe lung disease is defined in the same manner as in the CCI [11]. Each of the variables contributes 1 point and the FCI is a summation of these points; thus, the FCI value ranges from 0 to 3 .

2.5. Statistical Analysis. Numerical variables are summarized by median and range and categorical variables are described by count and relative frequency (\%) of subjects in each category. Comparison of the distribution of categorical variables in the different groups was performed with either Fisher's exact test or the $\chi^{2}$ test. OS was estimated using the KaplanMeier methodology. Uni- and multivariate Cox regression analyses were applied to assess factors affecting OS. Components with a $P$ value less than 0.05 in univariate analysis were included in the subsequent multivariate analysis.

These analyses were performed using PASW statistics 18.0.0 (WinWrap, IBM, New York, USA). Null hypotheses of no difference were rejected if $P$ values were less than 0.05 .

\section{Results}

3.1. Patient Characteristics. During the study period, a total of 159 patients aged 65 years or older were newly diagnosed with symptomatic MM. Among them, 22 patients were excluded because of a combined diagnosis of amyloidosis, early followup loss, or ASCT. Therefore, data from 127 patients were included in the analysis.

Table 2 shows baseline characteristics of patients at the time of diagnosis. The median overall survival of all patients was 34.1 months, and the median follow-up duration for the surviving patients was 46 months. The median age of the patients was 71 years and $26.8 \%$ of the patients were aged 75 years or older. Performance status was evaluated by ECOG status. In FCI, performance status was assessed by KPS, and, by definition, ECOG grade 2 is interchangeable with KPS grade 70 [19-21].

The prevalence of comorbidity at the time of diagnosis was $48.8 \%$ (Table 3 ). The most frequent comorbid condition was diabetes without end organ damage $(n=24,18.9 \%)$. The median CCI score, including age points, was 3 (range: 2 to 13). The CCI score was divided into two groups based on the median score; CCI scores of 2-3 were classified as the low CCI score group, and CCI scores $\geq 4$ were classified as the high CCI score group. In this study, 53 and 74 patients belonged to the low and high CCI score groups, respectively. When using the FCI classification, 59, 54, and 12 patients belonged to the 0,1 , and 2-3 score groups, respectively.

3.2. Host Factors and Comorbidity Indices as Prognostic Factors. We analyzed the impact of host factors, such as age, sex, performance status, and each of the comorbidities on OS (Table 4). The results showed that all of the factors constituting FCI, such as performance status, chronic lung disease, and eGFR, were significant for OS. In addition, any tumor, metastatic solid tumor, cerebrovascular disease, and ISS each had statistical significance. When multivariate analysis was conducted with these factors, only performance 
TABle 1: Definitions of the Charlson and Freiburg comorbidity indices.

\begin{tabular}{|c|c|c|}
\hline Weight & Condition & Definition \\
\hline \multirow{11}{*}{1} & & Charlson comorbidity index \\
\hline & Myocardial infarct & Hospitalization and electrocardiographic and/or enzyme change \\
\hline & Congestive heart failure & $\begin{array}{l}\text { Exertional or paroxysmal nocturnal dyspnea and responded symptomatically (or on } \\
\text { physical examination) to digitalis, diuretics, or afterload reducing agents }\end{array}$ \\
\hline & Peripheral vascular disease & $\begin{array}{l}\text { Intermittent claudication or prior bypass for arterial insufficiency; gangrene or acute arterial } \\
\text { insufficiency; untreated thoracic or abdominal aneurysm }(\geq 6 \mathrm{~cm})\end{array}$ \\
\hline & Cerebrovascular disease & Cerebrovascular accident with minor or no residual and transient ischemic attacks \\
\hline & Dementia & Chronic cognitive deficit \\
\hline & Chronic pulmonary disease & $\begin{array}{l}\text { Moderate: dyspneic with slight activity, with or without treatment, and dyspneic with } \\
\text { moderate activity despite treatment; Severe: dyspneic at rest, despite treatment, requires } \\
\text { constant oxygen; } \mathrm{CO}_{2} \text { retention and a baseline } \mathrm{PO}_{2} \text { below } 50 \text { torr }\end{array}$ \\
\hline & Connective tissue disease & SLE, PM, MCTD, polymyalgia rheumatic, and moderate to severe RA \\
\hline & Ulcer disease & Required treatment for ulcer disease, including bleeding from ulcers \\
\hline & Mild liver disease & Cirrhosis without portal hypertension or chronic hepatitis \\
\hline & Diabetes & $\begin{array}{l}\text { Mild: treated with insulin or oral hypoglycemics, but not with diet alone. Moderate: previous } \\
\text { hospitalizations for ketoacidosis, hyperosmolar coma, or/and those with juvenile onset or } \\
\text { brittle diabetics }\end{array}$ \\
\hline \multirow{6}{*}{2} & Hemiplegia & $\begin{array}{l}\text { Dense hemiplegia or paraplegia, as a result of either a cerebrovascular accident or other } \\
\text { conditions }\end{array}$ \\
\hline & Moderate or severe renal disease & Severe: on dialysis, had a transplant, and with uremia. Moderate: serum creatinine $>3 \mathrm{mg} \%$ \\
\hline & Diabetes with end organ damage & Severe: with retinopathy, neuropathy, or nephropathy \\
\hline & Any tumor & Solid tumors without documented metastases, but initially treated in the last 5 years \\
\hline & Leukemia & AML, CML, ALL, CLL, and PV \\
\hline & Lymphoma & HD, lymphosarcoma, WM, myeloma, and other lymphomas \\
\hline 3 & Moderate or severe liver disease & $\begin{array}{l}\text { Severe: cirrhosis, portal hypertension, and a history of variceal bleeding. Moderate: cirrhosis } \\
\text { with portal hypertension, but without history of variceal bleeding }\end{array}$ \\
\hline \multirow{2}{*}{6} & Metastatic solid tumor & Metastatic solid tumors \\
\hline & AIDS & Define or probable AIDS (i.e., AIDS related complex) \\
\hline \multirow{4}{*}{1} & & Freiburg comorbidity index \\
\hline & Renal impairment & $\mathrm{eGFR}_{\mathrm{MDRD}} \leq 30 \mathrm{~mL} / \mathrm{min} / 1.73 \mathrm{~m}^{2}$ \\
\hline & Performance status & Karnofsky performance status (KPS) score $\leq 70$ \\
\hline & Moderate or severe lung disease & Same as CCI \\
\hline
\end{tabular}

Abbreviations: SLE, systemic lupus erythematous; PM, polymyositis; MCTD, mixed connective tissue disease; RA, rheumatoid arthritis; AML, acute myelogenous leukemia; CML, chronic myelogenous leukemia; ALL, acute lymphocytic leukemia; CLL, acute lymphocytic leukemia; PV, polycythemia vera; HD, Hodgkin disease; WM, Waldenstrom's macroglobulinemia; AIDS, acquired immune deficiency syndrome; eGFR, estimated glomerular filtration rate; MDRD, modification of diet in renal disease; CCI, Charlson comorbidity index.

status and previous cancer history (regardless of metastasis) remained significant.

Figures 1, 2, 3, and 4 show Kaplan-Meier survival curves for both comorbidity indices. FCI effectively predicted the OS of the three different groups $(P<0.001)$. The median survival times were 55.0 months, 29.5 months, and 19.5 months for FCI scores of 0,1 , and 2-3, respectively. Although the $P$ value was not statistically significant, CCI also distinguished between the two score groups for OS (44.8 months versus 34.7 months, $P=0.059)$. However, OS based on CCI without age points did not demonstrate clinical relevance $(P=0.147)$. In contrast,
FCI was significant in subgroup analysis for age groups (6574 and $\geq 75$ years; $P<0.001$ and 0.04 , resp.)

3.3. Serious Adverse Events. We defined serious adverse events as grade $\geq 4$ for hematologic adverse events and grade $\geq 3$ for nonhematologic adverse events, according to NCICTC version 4.0. The most frequent serious adverse event was infection $(n=35,30.0 \%)$ followed by neutropenia and anemia ( $n=15,12.7 \%$ for both). Grade $\geq 3$ nonhematologic adverse events occurred in $50 \%$ of patients, whereas grade $\geq 4$ hematologic adverse events occurred in $22.9 \%$ of patients. 
TABLE 2: Baseline characteristics $(n=127)$.

\begin{tabular}{|c|c|c|}
\hline Characteristics & $N(\%)$ & Median (range) \\
\hline Age, years & & $71(65-92)$ \\
\hline 65-74 & $93(73.2 \%)$ & \\
\hline$\geq 75$ & $34(26.8 \%)$ & \\
\hline \multicolumn{3}{|l|}{ Sex } \\
\hline Male & $62(48.8 \%)$ & \\
\hline Female & $65(51.2 \%)$ & \\
\hline \multicolumn{3}{|l|}{ ECOG } \\
\hline $0-1$ & $74(58.3 \%)$ & \\
\hline$\geq 2$ & $52(40.9 \%)$ & \\
\hline Unknown & $1(0.8 \%)$ & \\
\hline \multicolumn{3}{|l|}{ Durie-Salmon stage } \\
\hline 1 & $10(7.9 \%)$ & \\
\hline 2 & $28(22.0 \%)$ & \\
\hline 3 & $89(70.1 \%)$ & \\
\hline \multicolumn{3}{|l|}{ International staging system } \\
\hline 1 & $23(18.1 \%)$ & \\
\hline 2 & $51(40.2 \%)$ & \\
\hline 3 & $47(37.0 \%)$ & \\
\hline Unknown & $6(4.7 \%)$ & \\
\hline \multicolumn{3}{|l|}{ Lytic bone lesion } \\
\hline Yes & $112(88.2 \%)$ & \\
\hline No & $14(11.0 \%)$ & \\
\hline Hemoglobin (g/dL) & & $9.66(5.20-16.30)$ \\
\hline$<10$ & $75(59.1 \%)$ & \\
\hline$\geq 10$ & $52(40.9 \%)$ & \\
\hline Platelets $\left(\times 10^{9} / \mathrm{L}\right)$ & & $195(44-484)$ \\
\hline$<100$ & $11(8.7 \%)$ & \\
\hline$\geq 100$ & $117(91.3 \%)$ & \\
\hline Plasma cells in bone marrow (\%) & & $43.88(1.10-100)$ \\
\hline$\geq 40$ & $58(45.7 \%)$ & \\
\hline$<40$ & $65(51.2 \%)$ & \\
\hline Serum calcium (mg/dL) & & $9.35(7.00-15.70)$ \\
\hline$>11.5$ & $11(8.7 \%)$ & \\
\hline$\leq 11.5$ & $115(90.6 \%)$ & \\
\hline Serum albumin $(\mathrm{mg} / \mathrm{dL})$ & & $3.28(1.70-4.80)$ \\
\hline$\leq 3.5$ & $85(66.9 \%)$ & \\
\hline$>3.5$ & $42(33.1 \%)$ & \\
\hline $\mathrm{eGFR}\left(\mathrm{mL} / \mathrm{min} / 1.73 \mathrm{~m}^{2}\right)$ & & $66.60(5.90-170.5)$ \\
\hline$>30$ & $112(88.2 \%)$ & \\
\hline$\leq 30$ & $15(11.8 \%)$ & \\
\hline \multicolumn{3}{|l|}{ Serum LD } \\
\hline >UNL & $27(21.3 \%)$ & \\
\hline$\leq \mathrm{UNL}$ & $74(58.3 \%)$ & \\
\hline \multicolumn{3}{|l|}{ Initial chemotherapy regimen } \\
\hline Conventional (CP, MP, and others) & $92(78.0 \%)$ & \\
\hline Novel agents (imid, bortezomib-based) & $26(22.0 \%)$ & \\
\hline \multicolumn{3}{|l|}{ Treatment } \\
\hline Chemotherapy & $118(92.9 \%)$ & \\
\hline No chemotherapy & $9(7.1 \%)$ & \\
\hline
\end{tabular}

ECOG, eastern cooperative oncology group; eGFR, estimated glomerular filtration rate; LD, lactate dehydrogenase; UNL, upper normal limit; CP, cyclophosphamide and prednisolone; MP, melphalan and prednisolone. 


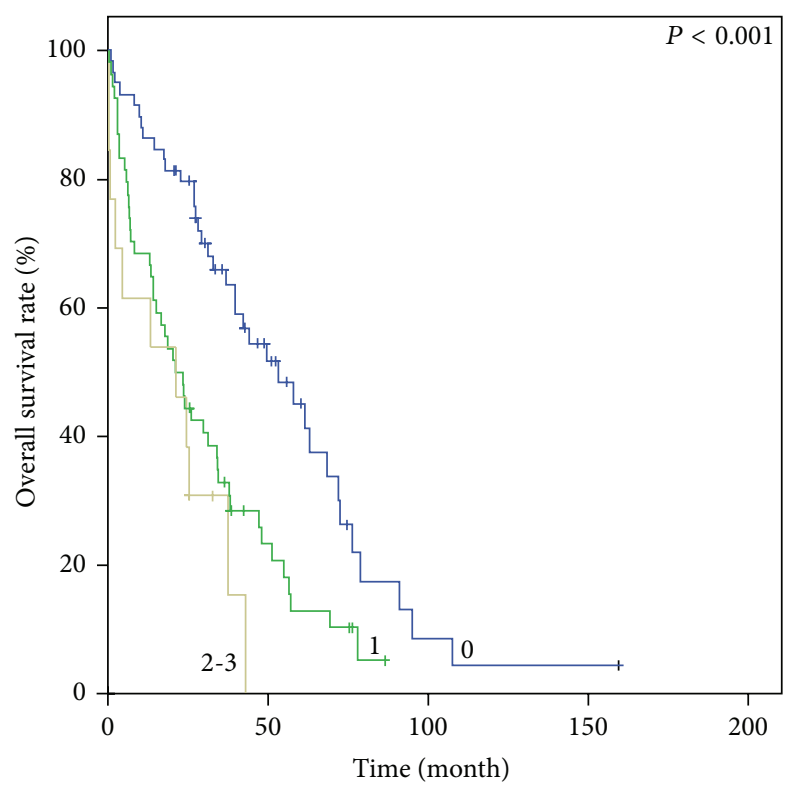

FIGURE 1: Kaplan-Meier survival curves of comorbidity index score groups. OS according to FCI.

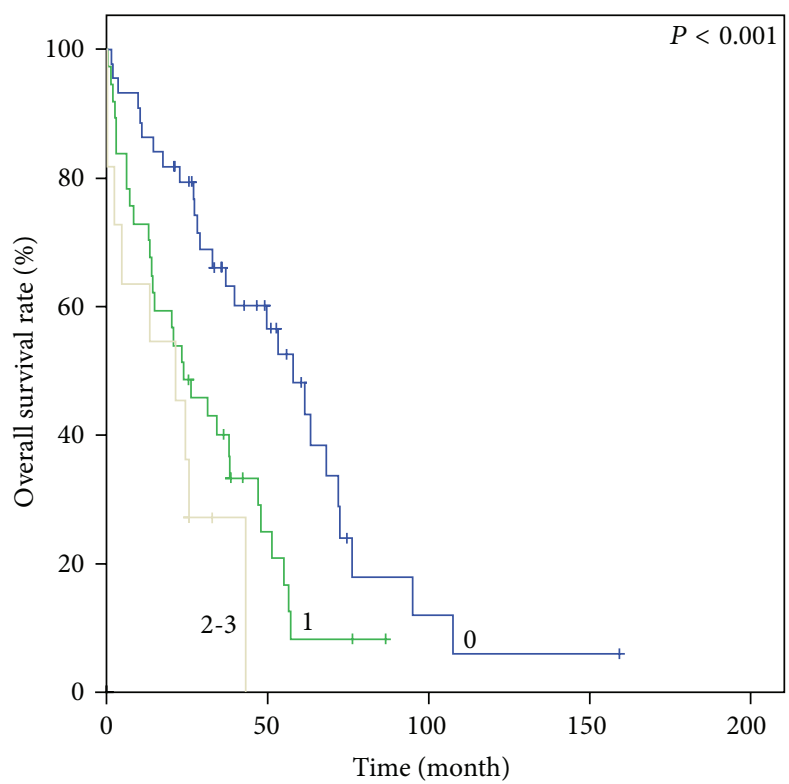

FIGURE 2: Kaplan-Meier survival curves of comorbidity index score groups. OS according to FCI in patients aged 65-74 years.

Grade 5 adverse events due to any cause occurred in $6.8 \%$ of the patients, as shown in Table 5.

\section{Discussion}

This study assessed comorbidities at diagnosis of MM, the impact of host factors on overall survival, and compared CCI and FCI as prognostic factors in newly diagnosed elderly patients.
Univariate analysis revealed that performance status, ISS, and several comorbid conditions such as chronic lung disease, azotemia $\left(e G F R<30 \mathrm{~mL} / \mathrm{min} / 1.73 \mathrm{~m}^{2}\right)$, presence of any tumor, metastatic solid tumor, and cerebrovascular disease were significant factors. However, azotemia as defined by CCI (serum creatinine $\geq 3 \mathrm{mg} / \mathrm{mL}$ ) was not a prognostic factor. In multivariate analysis, azotemia, as defined by impaired eGFR or chronic lung disease, was not shown to 


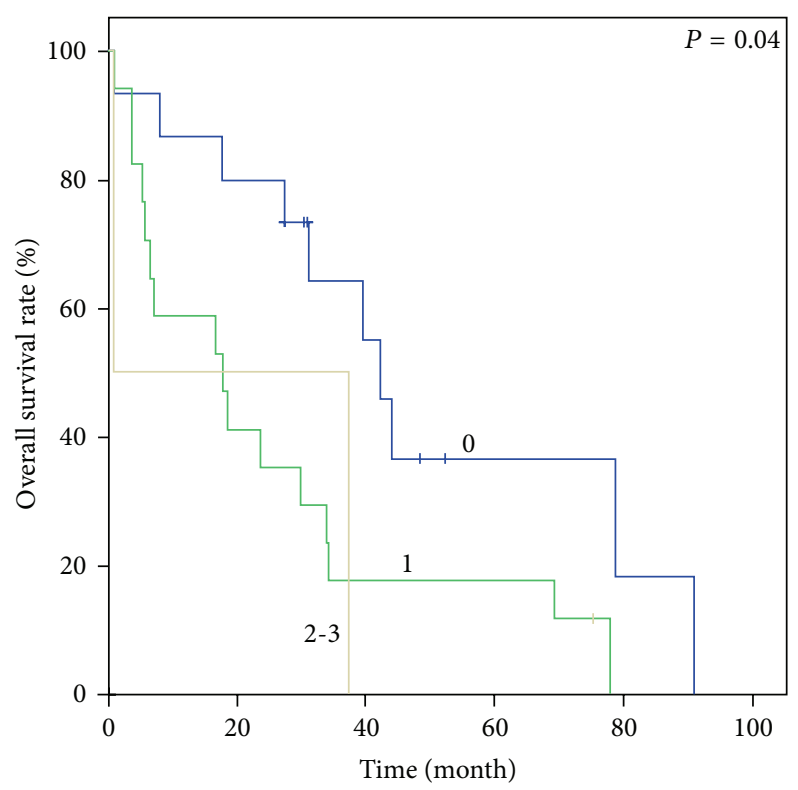

FIGURE 3: Kaplan-Meier survival curves of comorbidity index score groups. OS according to FCI in patients aged $\geq 75$ years.

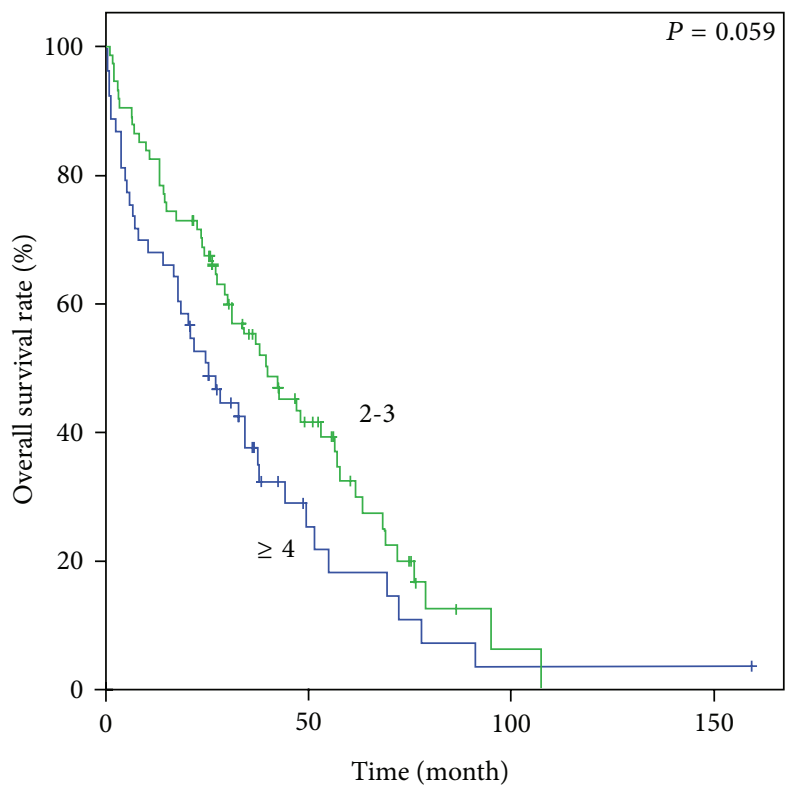

FIGURE 4: Kaplan-Meier survival curves of comorbidity index score groups. OS according to CCI.

be a significant risk factor in our study. In contrast, a history of cancer, regardless of whether metastasis occurred, was the strongest prognostic factor for elderly patients with myeloma. Unfortunately, use of novel agents over conventional drugs did not significantly improve OS, although this might reflect the relatively short period of use of novel agents.

Although two components of FCI-renal impairment and moderate or severe lung disease failed to demonstrate significance in multivariate analysis, when we compared both comorbidity indices and overall survival, the FCI showed a greater ability to separate OS among the three score groups $(P<0.001)$. The CCI score including age points was not statistically significant but was still valuable and superior to the CCI without age points. As briefly mentioned above, the CCI score without age points did not discriminate for OS.

FCI provides a clear definition of each component and all three components were statistically significant, at least in univariate analysis. In contrast, CCI is more subjective and 
TABLE 3: Prevalence of comorbidities according to the Charlson and Freiburg comorbidity indices and patient distribution according to comorbidity indices.

(a) Prevalence of comorbidities according to the Charlson comorbidity index.

\begin{tabular}{lcc}
\hline Comorbidity & Yes & No \\
\hline Myocardial infarct & $7(5.5 \%)$ & $120(94.5 \%)$ \\
Congestive heart failure & $7(5.5 \%)$ & $120(94.5 \%)$ \\
Peripheral vascular disease & $0(0 \%)$ & $127(100 \%)$ \\
Cerebrovascular disease & $6(4.7 \%)$ & $121(95.3 \%)$ \\
Dementia & $0(0 \%)$ & $127(100 \%)$ \\
Chronic lung disease & $14(11.0 \%)$ & $113(89.0 \%)$ \\
Connective tissue disease & $2(1.6 \%)$ & $125(98.4 \%)$ \\
Ulcer disease & $8(6.3 \%)$ & $119(93.7 \%)$ \\
Mild liver disease & $2(1.6 \%)$ & $125(98.4 \%)$ \\
DM & $24(18.9 \%)$ & $103(81.1 \%)$ \\
Hemiplegia & $6(4.7 \%)$ & $121(95.3 \%)$ \\
Moderate to severe renal disease & $1(0.8 \%)$ & $126(99.2 \%)$ \\
DM with end organ damage & $0(0 \%)$ & $127(100 \%)$ \\
Any tumor & $8(6.3 \%)$ & $119(93.7 \%)$ \\
Leukemia & $0(0 \%)$ & $127(100 \%)$ \\
Lymphoma & $0(0 \%)$ & $127(100 \%)$ \\
Moderate to severe liver disease & $2(1.6 \%)$ & $125(98.4 \%)$ \\
Metastatic solid tumor & $2(1.6 \%)$ & $125(98.4 \%)$ \\
AIDS & $0(0 \%)$ & $127(100 \%)$ \\
\hline DM, & &
\end{tabular}

DM, diabetes mellitus; AIDS, acquired immune deficiency syndrome.

(b) Prevalence of comorbidities according to the Freiburg comorbidity index.

\begin{tabular}{lcc}
\hline Component & Yes & No \\
\hline $\begin{array}{l}\text { Renal impairment } \\
\left(\mathrm{eGFR} \leq 30 \mathrm{~mL} / \mathrm{min} / 1.73 \mathrm{~m}^{2}\right)\end{array}$ & $15(11.8 \%)$ & $112(88.2 \%)$ \\
Performance status $(\mathrm{KPS} \leq 70)$ & $52(40.9 \%)$ & $74(58.3 \%)$ \\
Moderate or severe lung disease & $14(11.0 \%)$ & $113(89.0 \%)$ \\
\hline
\end{tabular}

eGFR, estimated glomerular filtration rate; KPS, Karnofsky performance status.

(c) Patient distribution according to comorbidity indices.

\section{(I) CCI total}

(comorbidity scores without age points)$$
0
$$$$
1
$$$$
2
$$$$
3
$$$$
4
$$$$
6
$$$$
8
$$$$
1(0.8 \%)
$$

(II) CCI total

(comorbidity scores with age points)

$$
2
$$$$
3
$$

(c) Continued.

\begin{tabular}{lc}
\hline 5 & $9(7.1 \%)$ \\
6 & $5(3.9 \%)$ \\
7 & $6(4.7 \%)$ \\
9 & $1(0.8 \%)$ \\
13 & $1(0.8 \%)$ \\
(III) CCI score group (with age points) & \\
Low $(2-3)$ & $53(41.7 \%)$ \\
High $(\geq 4)$ & $74(58.3 \%)$ \\
(IV) FCI & \\
0 & $59(46.5 \%)$ \\
1 & $54(425 \%)$ \\
2 & $12(9.4 \%)$ \\
3 & $1(0.8 \%)$ \\
Unknown & $1(0.8 \%)$ \\
\hline CCI, Charlson comorbidity index; FCI, Freiburg comorbidity index.
\end{tabular}

only 4 among 19 conditions were significant. Most importantly, at the present time, CCI does not have any standard cut-off value. Various studies have divided CCI scores into groups of $0,1-2$, and $\geq 3 ; 0,1$, and $\geq 2$; or 0 and $\geq 1$. Some studies included an age point, but others did not [11-14]. These variations might explain why FCI is more predictable than CCI.

Moreover, FCI is also very simple to apply. FCI consists of performance status, moderate or severe lung disease, and azotemia, and each of these factors is worth 1 point. The FCI score is, therefore, a simple summation of these three factors. In contrast, CCI consists of 19 comorbid conditions, and, within the same disease, scores are weighted based on severity ranging from 1 to 6 points. In addition, an age point is calculated and added to the CCI score. Comorbidity definitions frequently use a symptomatic grade.

In this study, all 118 patients who were treated with chemotherapy received a full dose of chemotherapeutic agents as scheduled. Interestingly, the profile for serious adverse events showed that treatment was relatively safe and adverse events were easily controllable. In fact, since the approval of various novel agents, clinical outcomes such as survival and toxicity profiles have improved in transplant-ineligible elderly patients with multiple myeloma [22-27]. Thus, we propose that the full dose of chemotherapy might be tolerated, regardless of the presence of comorbid conditions, even though this is against the recommendation that chemotherapy dose reduction is required for patients 75 years or older or those with cardiac, pulmonary, hepatic, renal, or neurologic dysfunctions [5]. Furthermore, this finding might be important evidence for preventing chemotherapy dose reduction because of physician bias.

There are some limitations in this study. First, the followup duration was short and the sample size was small. Second, this is a retrospective single center study. Third, there were no patients with peripheral vascular disease, dementia, DM with end organ damage, or AIDS. Despite these limitations, this study successfully applied the FCI and the CCI to newly 
TABLE 4: Univariate and multivariate Cox's regression analysis for overall survival.

\begin{tabular}{|c|c|c|c|c|c|c|}
\hline & \multicolumn{3}{|c|}{ Univariate analysis } & \multicolumn{3}{|c|}{ Multivariate analysis } \\
\hline & $P$ value & HR & $95 \% \mathrm{CI}$ & $P$ value & HR & $95 \% \mathrm{CI}$ \\
\hline ECOG & 0.002 & 1.951 & $1.287-2.958$ & 0.009 & 1.890 & $1.176-3.038$ \\
\hline \multicolumn{7}{|l|}{$0-1$} \\
\hline \multicolumn{7}{|l|}{$\geq 2$} \\
\hline Chronic lung disease & 0.028 & 1.941 & $1.073-3.510$ & 0.300 & 1.425 & $0.730-2.778$ \\
\hline \multicolumn{7}{|l|}{ Yes } \\
\hline \multicolumn{7}{|l|}{ No } \\
\hline $\mathrm{eGFR}\left(\mathrm{mL} / \mathrm{min} / 1.73 \mathrm{~m}^{2}\right)$ & 0.012 & 2.139 & $1.183-3.869$ & 0.228 & 1.515 & $0.771-2.976$ \\
\hline \multicolumn{7}{|l|}{$>30$} \\
\hline \multicolumn{7}{|l|}{$\leq 30$} \\
\hline Any tumor & 0.001 & 3.513 & $1.678-7.356$ & 0.003 & 3.717 & $1.617-8.554$ \\
\hline \multicolumn{7}{|l|}{ Yes } \\
\hline \multicolumn{7}{|l|}{ No } \\
\hline Metastatic solid tumor & $<0.001$ & 44.034 & $8.449-229.485$ & $<0.001$ & 85.847 & $14.628-503.822$ \\
\hline \multicolumn{7}{|l|}{ Yes } \\
\hline \multicolumn{7}{|l|}{ No } \\
\hline Cerebrovascular disease & 0.016 & 3.064 & $1.228-7.641$ & 0.210 & 1.855 & $0.706-4.887$ \\
\hline \multicolumn{7}{|l|}{ Yes } \\
\hline \multicolumn{7}{|l|}{ No } \\
\hline International staging system & 0.024 & & & 0.250 & & \\
\hline \multicolumn{7}{|l|}{1} \\
\hline \multicolumn{7}{|l|}{2} \\
\hline \multicolumn{7}{|l|}{3} \\
\hline Age, years & 0.339 & 1.242 & $0.796-1.938$ & & & \\
\hline \multicolumn{7}{|l|}{$65-74$} \\
\hline \multicolumn{7}{|l|}{$\geq 75$} \\
\hline Sex & 0.692 & 0.920 & $0.610-1.388$ & & & \\
\hline \multicolumn{7}{|l|}{ Male } \\
\hline Female & & & & & & \\
\hline Durie-Salmon stage & 0.242 & & & & & \\
\hline 1 & & & & & & \\
\hline 2 & & & & & & \\
\hline 3 & & & & & & \\
\hline Myocardial infarct & 0.146 & 1.783 & $0.818-3.884$ & & & \\
\hline Yes & & & & & & \\
\hline No & & & & & & \\
\hline Congestive heart failure & 0.581 & 1.329 & $0.484-3.644$ & & & \\
\hline Yes & & & & & & \\
\hline No & & & & & & \\
\hline Connective tissue disease & 0.302 & 0.047 & $0.000-15.543$ & & & \\
\hline Yes & & & & & & \\
\hline No & & & & & & \\
\hline Ulcer disease & 0.196 & 0.571 & $0.244-1.334$ & & & \\
\hline Yes & & & & & & \\
\hline No & & & & & & \\
\hline Mild liver disease & 0.262 & 2.246 & $0.545-9.249$ & & & \\
\hline Yes & & & & & & \\
\hline No & & & & & & \\
\hline $\mathrm{DM}$ & 0.256 & 0.718 & $0.405-1.272$ & & & \\
\hline
\end{tabular}


TABLE 4: Continued.

\begin{tabular}{|c|c|c|c|c|c|c|}
\hline & \multicolumn{3}{|c|}{ Univariate analysis } & \multicolumn{3}{|c|}{ Multivariate analysis } \\
\hline & $P$ value & HR & $95 \% \mathrm{CI}$ & $P$ value & HR & $95 \% \mathrm{CI}$ \\
\hline \multicolumn{7}{|l|}{ Yes } \\
\hline \multicolumn{7}{|l|}{ No } \\
\hline Hemiplegia & 0.081 & 0.353 & $0.110-1.135$ & & & \\
\hline \multicolumn{7}{|l|}{ Yes } \\
\hline \multicolumn{7}{|l|}{ No } \\
\hline Moderate to severe renal disease & 1.000 & 1.000 & $0.000-4.271 E 9$ & & & \\
\hline \multicolumn{7}{|l|}{ Yes } \\
\hline \multicolumn{7}{|l|}{ No } \\
\hline Moderate-severe liver disease & 0.302 & 2.100 & $0.514-8.583$ & & & \\
\hline \multicolumn{7}{|l|}{ Yes } \\
\hline \multicolumn{7}{|l|}{ No } \\
\hline Initial chemotherapy regimen & 0.844 & 1.058 & $0.603-1.858$ & & & \\
\hline \multicolumn{7}{|l|}{ Conventional agents } \\
\hline \multicolumn{7}{|l|}{ Novel agent } \\
\hline CCI & 0.061 & 0.677 & $0.450-1.018$ & & & \\
\hline \multicolumn{7}{|l|}{$2-3$} \\
\hline \multicolumn{7}{|l|}{$\geq 4$} \\
\hline FCI & $<0.001$ & & & & & \\
\hline \multicolumn{7}{|l|}{0} \\
\hline \multicolumn{7}{|l|}{1} \\
\hline $2-3$ & & & & & & \\
\hline
\end{tabular}

HR, hazard ratio; CI, confidence interval; ECOG, eastern cooperative oncology group; eGFR, estimated glomerular filtration rate; DM, diabetes mellitus; CCI, Charlson comorbidity index; FCI, Freiburg comorbidity index.

TABLE 5: Serious adverse events (AEs).

\begin{tabular}{|c|c|c|}
\hline & \multicolumn{2}{|c|}{$N(\%)$} \\
\hline & Grade $0-3$ & Grade $4-5$ \\
\hline \multicolumn{3}{|l|}{ Hematologic AE } \\
\hline Anemia & $103(87.3 \%)$ & $15(12.7 \%)$ \\
\hline Neutropenia & $103(87.3 \%)$ & $15(12.7 \%)$ \\
\hline Thrombocytopenia & $114(92.2 \%)$ & $4(7.8 \%)$ \\
\hline \multirow[t]{2}{*}{ Febrile neutropenia } & $113(90.8 \%)$ & $5(9.2 \%)$ \\
\hline & Grade $0-2$ & Grade $3-5$ \\
\hline \multicolumn{3}{|l|}{ Nonhematologic AE } \\
\hline Infection & $83(70.0 \%)$ & $35(30.0 \%)$ \\
\hline Diarrhea/constipation & $105(89.0 \%)$ & $13(11.0 \%)$ \\
\hline Fatigue & $117(90.7 \%)$ & $11(9.3 \%)$ \\
\hline Sensory neuropathy & $108(91.5 \%)$ & $10(8.5 \%)$ \\
\hline Nausea/vomiting & $111(94.1 \%)$ & $7(5.9 \%)$ \\
\hline Azotemia & $112(94.9 \%)$ & $6(5.1 \%)$ \\
\hline Grade $\geq 4$ hematologic $\mathrm{AE}$ & \multicolumn{2}{|c|}{$27(22.9 \%)$} \\
\hline Grade $\geq 3$ nonhematologic AE & \multicolumn{2}{|c|}{$59(50.0 \%)$} \\
\hline Grade 5 AE & \multicolumn{2}{|c|}{$8(6.8 \%)$} \\
\hline
\end{tabular}


diagnosed elderly multiple myeloma patients and revealed the superiority of FCI to CCI in predicting OS.

\section{Conclusions}

In this study, approximately $50 \%$ of elderly patients with newly diagnosed multiple myeloma had at least one comorbid disease at the time of diagnosis. Among host factors tested, performance status and a history of malignancy were the most important prognostic factors. The Freiburg comorbidity index is very simple to use and predicts overall survival better than the Charlson comorbidity index.

\section{Conflict of Interests}

The authors declare that there is no conflict of interests regarding the publication of this paper.

\section{References}

[1] S. Y. Kristinsson, O. Landgren, P. W. Dickman, Å. R. Derolf, and M. Björkholm, "Patterns of survival in multiple myeloma: a population-based study of patients diagnosed in Sweden from 1973 to 2003," Journal of Clinical Oncology, vol. 25, no. 15, pp. 1993-1999, 2007.

[2] M. Kleber, G. Ihorst, B. Deschler et al., "Detection of renal impairment as one specific comorbidity factor in multiple myeloma: multicenter study in 198 consecutive patients," European Journal of Haematology, vol. 83, no. 6, pp. 519-527, 2009.

[3] M. Kleber, G. Ihorst, J. Udi, B. Koch, R. Wsch, and M. Engelhardt, "Prognostic risk factor evaluation in patients with relapsed or refractory multiple myeloma receiving lenalidomide treatment: analysis of renal function by eGFR and of additional comorbidities by comorbidity appraisal," Clinical Lymphoma, Myeloma \& Leukemia, vol. 12, no. 1, pp. 38-48, 2012.

[4] E. Eleftherakis-Papapiakovou, E. Kastritis, M. Roussou et al., "Renal impairment is not an independent adverse prognostic factor in patients with multiple myeloma treated upfront with novel agent-based regimens," Leukemia \& lymphoma, vol. 52, no. 12, pp. 2299-2303, 2011.

[5] A. Palumbo, S. Bringhen, H. Ludwig et al., "Personalized therapy in multiple myeloma according to patient age and vulnerability: a report of the European Myeloma Network (EMN)," Blood, vol. 118, no. 17, pp. 4519-4529, 2011.

[6] A. Nanda, M. Chen, M. H. Braccioforte, B. J. Moran, and A. V. D'Amico, "Hormonal therapy use for prostate cancer and mortality in men with coronary artery disease-induced congestive heart failure or myocardial infarction," The Journal of the American Medical Association, vol. 302, no. 8, pp. 866$873,2009$.

[7] J. A. Meyerhardt, P. J. Catalano, D. G. Haller et al., "Impact of diabetes mellitus on outcomes in patients with colon cancer," Journal of Clinical Oncology, vol. 21, no. 3, pp. 433-440, 2003.

[8] P. J. Goodwin, M. Ennis, K. I. Pritchard et al., "Fasting insulin and outcome in early-stage breast cancer: results of a prospective cohort study," Journal of Clinical Oncology, vol. 20, no. 1, pp. 42-51, 2002.

[9] G. R. Simon, M. Extermann, A. Chiappori et al., "Phase 2 trial of docetaxel and gefitinib in the first-line treatment of patients with advanced nonsmall-cell lung cancer (NSCLC) who are 70 years of age or older," Cancer, vol. 112, no. 9, pp. 2021-2029, 2008.
[10] F. Gay and A. Palumbo, "Management of older patients with multiple myeloma," Blood Reviews, vol. 25, no. 2, pp. 65-73, 2011.

[11] M. Kleber, G. Ihorst, M. Terhorst et al., "Comorbidity as a prognostic variable in multiple myeloma: comparative evaluation of common comorbidity scores and use of a novel MMcomorbidity score," Blood Cancer Journal, vol. 1, no. 9, article e35, 2011.

[12] T. S. Field, J. L. F. Bosco, M. N. Prout et al., "Age, comorbidity, and breast cancer severity: impact on receipt of definitive local therapy and rate of recurrence among older women with earlystage breast cancer," Journal of the American College of Surgeons, vol. 213, no. 6, pp. 757-765, 2011.

[13] M. L. Sorror, M. B. Maris, B. Storer et al., "Comparing morbidity and mortality of HLA-matched unrelated donor hematopoietic cell transplantation after nonmyeloablative and myeloablative conditioning: influence of pretransplantation comorbidities," Blood, vol. 104, no. 4, pp. 961-968, 2004.

[14] L. Labonté, T. Iqbal, M. A. Zaidi et al., "Utility of comorbidity assessment in predicting transplantation-related toxicity following autologous hematopoietic stem cell transplantation for multiple myeloma," Biology of Blood and Marrow Transplantation, vol. 14, no. 9, pp. 1039-1044, 2008.

[15] M. Offidani, L. Corvatta, C. Polloni et al., "Assessment of vulnerability measures and their effect on survival in a reallife population of multiple myeloma patients registered at marche region multiple myeloma registry," Clinical Lymphoma, Myeloma and Leukemia, vol. 12, no. 6, pp. 423-432, 2012.

[16] W. R. Sperr, F. Wimazal, M. Kundi et al., "Comorbidity as prognostic variable in MDS: comparative evaluation of the HCT-CI and CCI in a core dataset of 419 patients of the Austrian MDS study group," Annals of Oncology, vol. 21, no. 1, Article ID mdp258, pp. 114-119, 2010.

[17] M. E. Charlson, P. Pompei, K. A. Ales, and C. R. MacKenzie, "A new method of classifying prognostic comorbidity in longitudinal studies: development and validation," Journal of Chronic Diseases, vol. 40, no. 5, pp. 373-383, 1987.

[18] A. S. Levey, L. A. Stevens, C. H. Schmid et al., "A new equation to estimate glomerular filtration rate," Annals of Internal Medicine, vol. 150, no. 9, pp. 604-612, 2009.

[19] I. de Kock, M. Mirhosseini, F. Lau et al., "Conversion of Karnofsky Performance Status (KPS) and Eastern Cooperative Oncology Group Performance Status (ECOG) to Palliative Performance Scale (PPS), and the interchangeability of PPS and KPS in prognostic tools," Journal of Palliative Care, vol. 29, no. 3, pp. 163-169, 2013.

[20] C. Ma, S. Bandukwala, D. Burman et al., "Interconversion of three measures of performance status: an empirical analysis," European Journal of Cancer, vol. 46, no. 18, pp. 3175-3183, 2010.

[21] M. M. Oken, R. H. Creech, and D. C. Tormey, "Toxicology and response criteria of the Eastern Cooperative Oncology Group," The American Journal of Clinical Oncology: Cancer Clinical Trials, vol. 5, no. 6, pp. 649-655, 1982.

[22] S. A. Ataergin, T. Kindwall-Keller, N. A. Berger, and H. M. Lazarus, "New generation pharmacotherapy in elderly multiple myeloma patients," Expert Opinion on Pharmacotherapy, vol. 10, no. 1, pp. 81-98, 2009.

[23] A. Palumbo, F. Davies, M. Kropff et al., "Consensus guidelines for the optimal management of adverse events in newly diagnosed, transplant-ineligible patients receiving melphalan and prednisone in combination with thalidomide (MPT) for the treatment of multiple myeloma," Annals of Hematology, vol. 89, no. 8, pp. 803-811, 2010. 
[24] A. A. Chanan-Khan, S. Lonial, D. Weber et al., "Lenalidomide in combination with dexamethasone improves survival and timeto-progression in patients $\geq 65$ years old with relapsed or refractory multiple myeloma," International Journal of Hematology, vol. 96, no. 2, pp. 254-262, 2012.

[25] J.-L. Harousseau and M. Dreyling, "Multiple myeloma: ESMO clinical practice guidelines for diagnosis, treatment and followup," Annals of Oncology, vol. 21, supplement 5, pp. v155-v157, 2010.

[26] A. Palumbo, M. Attal, and M. Roussel, "Shifts in the therapeutic paradigm for patients newly diagnosed with multiple myeloma: Maintenance therapy and overall survival," Clinical Cancer Research, vol. 17, no. 6, pp. 1253-1263, 2011.

[27] G. Barosi, G. Merlini, A. Billio et al., "SIE, SIES, GITMO evidence-based guidelines on novel agents (thalidomide, bortezomib, and lenalidomide) in the treatment of multiple myeloma," Annals of Hematology, vol. 91, no. 6, pp. 875-888, 2012. 


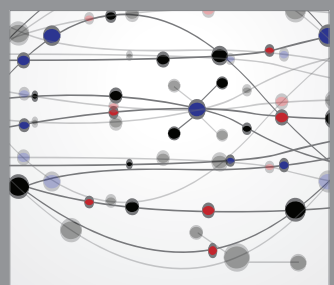

The Scientific World Journal
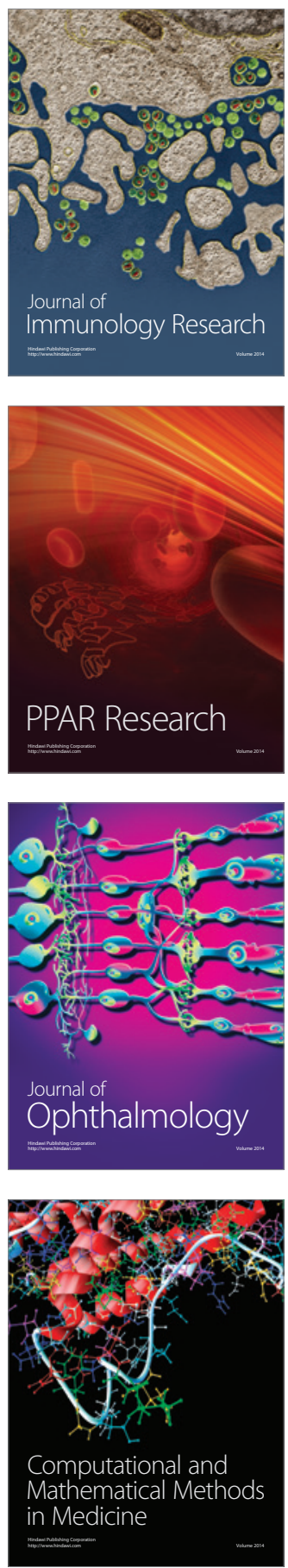

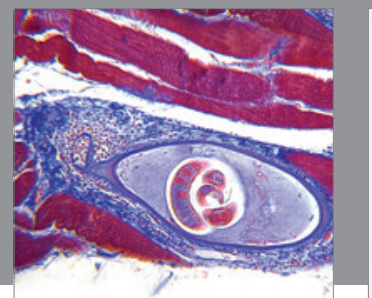

Gastroenterology

Research and Practice
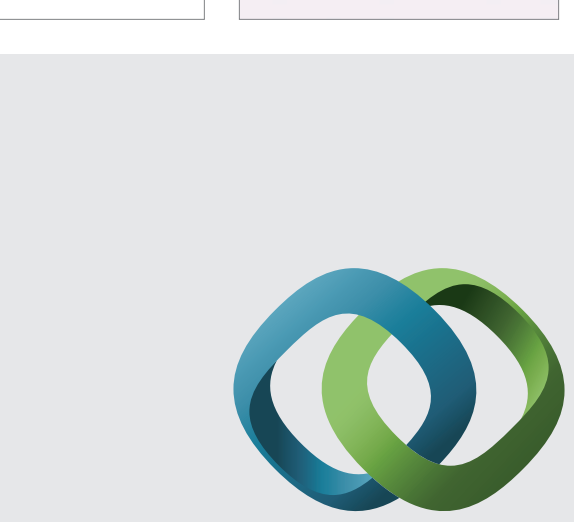

\section{Hindawi}

Submit your manuscripts at

http://www.hindawi.com
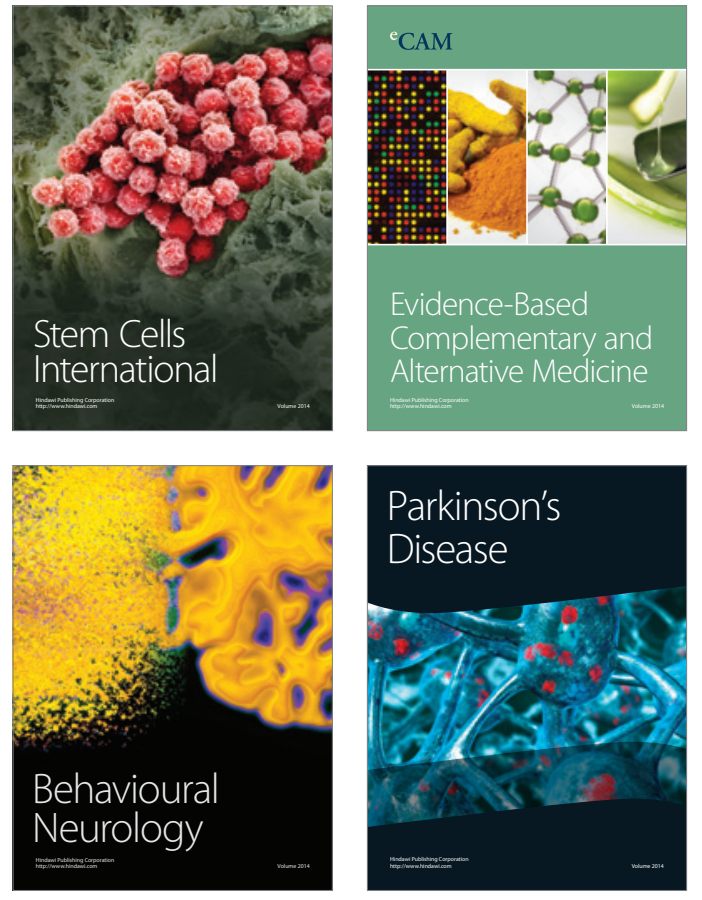
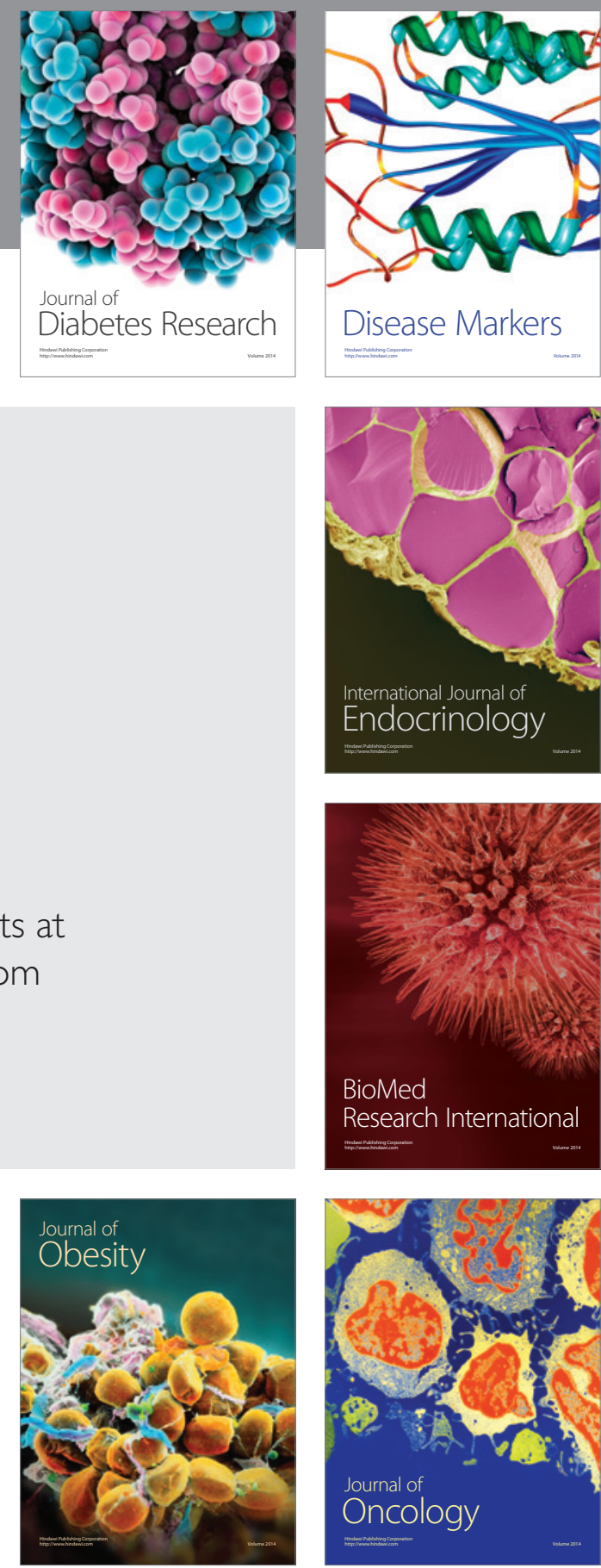

Disease Markers
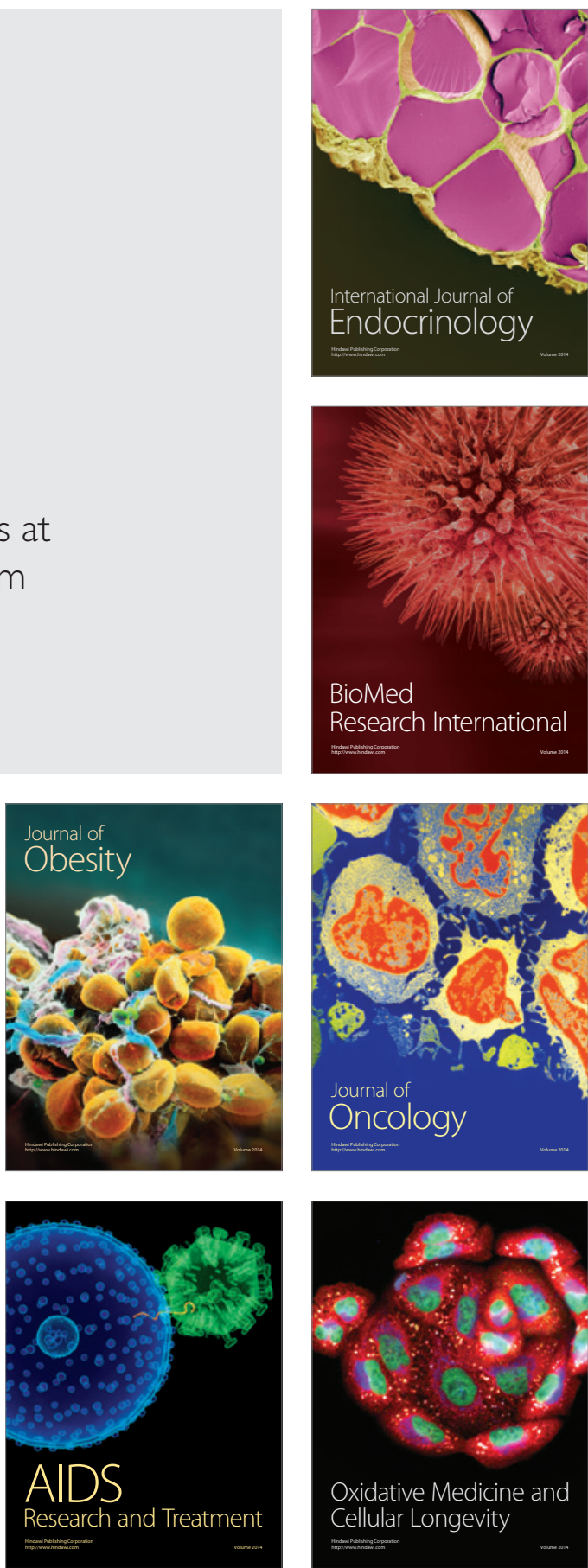